\title{
Epilepsy partialis continua with visual allesthesia
}

\author{
Mario F. Mendez $\cdot$ James W. Y. Chen
}

Received: 14 August 2008/Revised: 29 October 2008/Accepted: 19 November 2008/Published online: 25 February 2009

(C) The Author(s) 2009. This article is published with open access at Springerlink.com

\section{Sirs}

Visual allesthesia is a condition in which visual images are transposed from one half of the visual field to the other. The most common cause is an acute right parietal stroke, but allesthesia can result from other lesions and mechanisms. We describe the unusual case of a man with continuous transposition of his right visual field into his left due to epilepsia partialis continua.

A 57-year-old, right-handed man with a remote gunshot wound to the right parietal lobe presented with the complaint of seeing everything on his right-side moved to his left-side. He initially developed paroxysmal episodes that began with an awareness that visual objects were transposed from his right visual field to his left. The transposed objects were less distinct than the original, appeared further away, and involved background as well as foreground. The patient turned his eyes and his head to the left in order to capture the original left-sided images. The transposition persisted a few minutes after removal of the original visual stimulus and despite closing either or both eyes. About a minute into the transpositions, he experienced other sensory phenomena including a repetitive "clicking or crackling" sound on the left side and a numbness of his left hand. He would then recover with a residual "fogginess" and right-sided headache. The entire episodes usually

M. F. Mendez $(\bowtie) \cdot$ J. W. Y. Chen

Department of Neurology, Neurobehavior Unit (116AF),

V.A. Greater Los Angeles Healthcare System,

11301 Wilshire Blvd., Los Angeles, CA 90073, USA

e-mail: mmendez@ucla.edu

M. F. Mendez · J. W. Y. Chen

David Geffen School of Medicine, University of California,

Los Angeles, USA lasted 3-4 min, but, at time of presentation, the visual transposition had increased in frequency to the point of becoming continuous.

On past medical history, he had a penetrating head injury 18 years previously, post-traumatic seizures (simple motor involving his left upper extremity), mild glaucoma responsive to ophthalmic drops, and hearing loss. His epilepsy responded to phenytoin and then gabapentin, which he had discontinued 8 years previously. Afterwards, he continued with monthly episodes of left upper extremity numbness and weakness. Six months before presentation, he underwent MRI for evaluation of his hearing loss.

$\mathrm{He}$ underwent examination during the ongoing visual transpositions. He reported that the examiner, standing on his right side, was seen on his left. When the examiner moved items on the right, the patient reported the movements on the left. He was otherwise alert, attentive, and oriented with fluent speech, normal auditory comprehension, and normal memory testing. The rest of his examination was normal except for diminished left hemibody pinprick sensation, left hand astereognosia and agraphaesthesia, and a left upgoing toe.

After resolution of his visual transpositions, he showed left hemifield extinction on double simultaneous stimulation, but no hemispatial neglect on line bisection and visual search tasks. On visual examination, he had visual acuity of OD 20/25 and OS $20 / 30+2$ and a left superior arcuate visual field deficit in his right eye attributed to glaucoma rather than to his right parietal predominant encephalomalacia (Fig. 1).

The patient's presentation was consistent with partial simple seizures. On hospitalization, he had continuous clinical seizures lasting over an hour. Electroencephalogram taken on day 2 and 3 after hospitalization showed 
Fig. 1 Magnetic resonance axial flair images at lower (a) and higher (b) levels. There is a large area of encephalomalacia along the right parietal lobe, extending from the sensory strip to the precuneus. There is a significant amount of gliosis in the white matter, with some sparing of the overlying cortical ribbon. The temporal lobes, including the mesial temporal structures, are grossly normal. There is calvarial thinning from a prior craniotomy overlying the region of encephalomalacia.

Visual field examination (c, d). Right eye superior arcuate field defect, nasal step (a). Left eye beginning of inferior arcuate defect (b).

Electroencephalogram (EEG) at the beginning of the ictal buildup (e) and during the visual allesthesia (f). Ictal focal parietal-posterior temporaloccipital episodes. The EEG demonstrated phase reversal at $\mathrm{P} 4$ spreading to $\mathrm{T} 4$, localized to the right parieto-temporal cortex and lasting 179-320 s. The two EEGs were calibrated at $60 \mu \mathrm{V}$. A prior EEG, before development of the spells of visual allesthesia, had shown transient irregular medium voltages 3-7 cycles per second slowing in the right parietal region, but no clear cut epileptiform activity

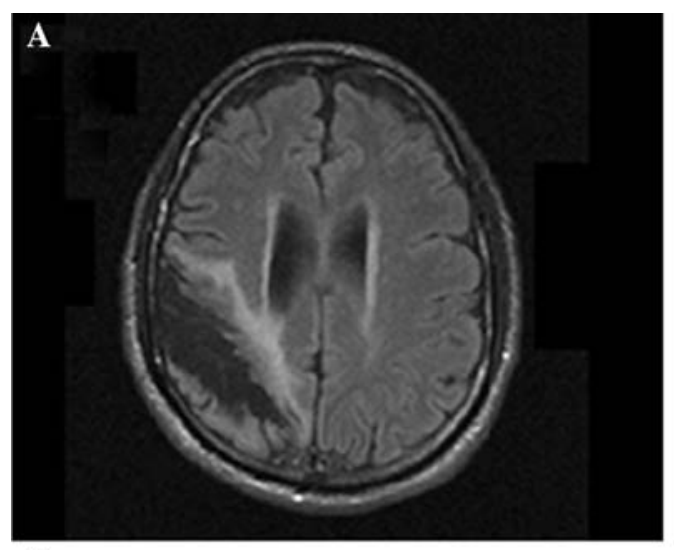

C

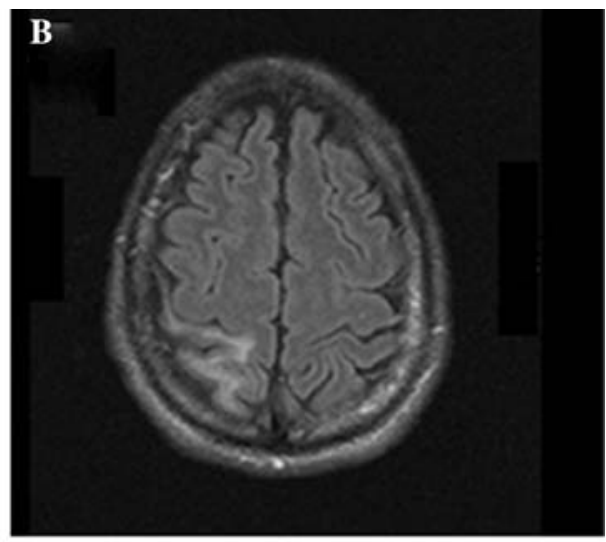

D

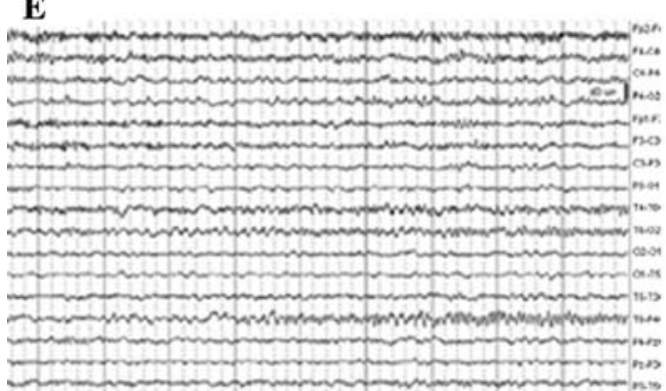

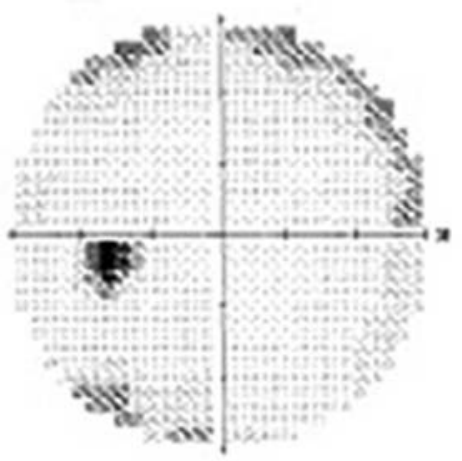

F
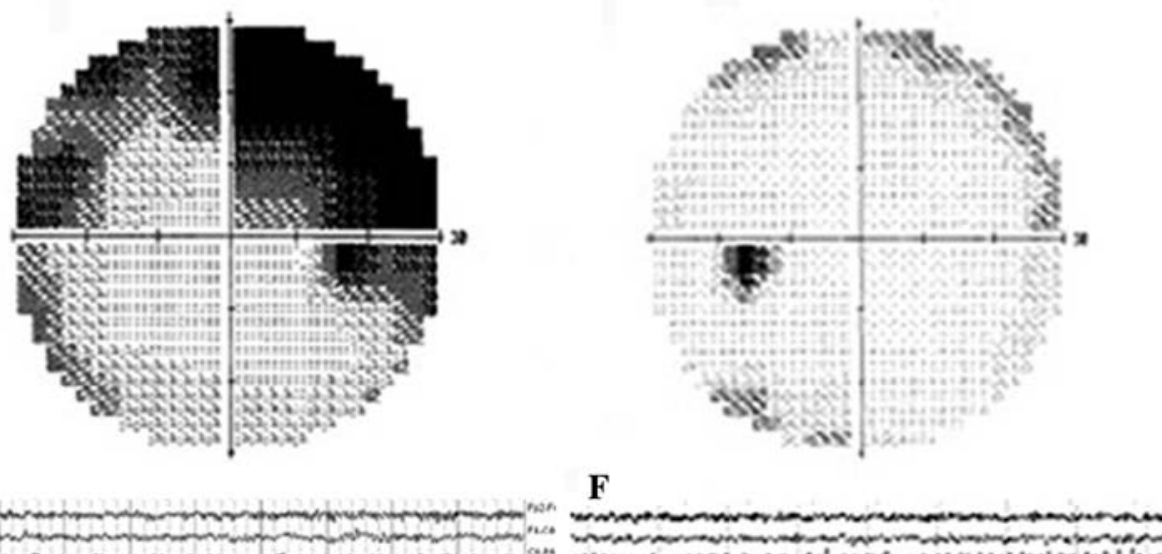

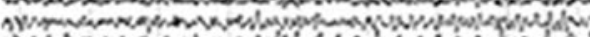

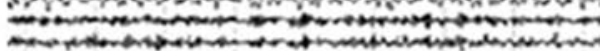

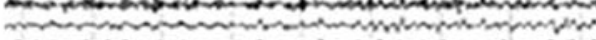

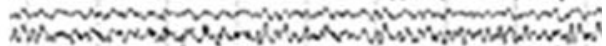

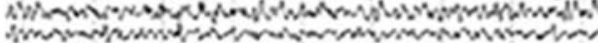

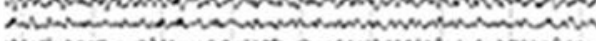

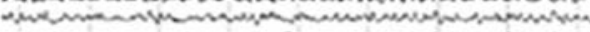

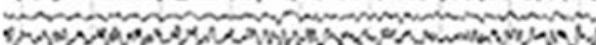

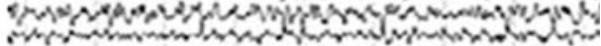

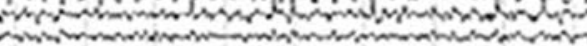

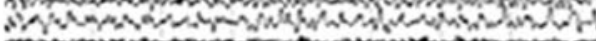

ictal focal episodes emanating from the right parieto-temporal region (Fig. 1). He was administered lorazepam $2 \mathrm{mg}$ IV and loaded with levetiracetam 1,500 mg IV. His seizures gradually subsided, and he was discharged with only occasional partial seizures on levetiracetam 2,000 mg BID, divalproex 1,500 $\mathrm{mg}$ BID, and gabapentin 1,200 mg QID.

This patient had ictal visual allesthesia, or the epileptogenic transfer of visual information from his right hemifield to his left hemifield [1, 2]. This was accompanied by palinopsia or the persistence of the transposed images [1]. Visual allesthesia usually results from right hemisphere lesions with left-sided neglect or extinction [2, 3]. In addition, investigators have reported ictal or post-ictal visual allesthesia in patients with right parieto-occipital or temporal-occipital arteriovenous malformations and in a patient with a right falcotentorial meningioma compressing the area around Brodmann's areas 18 and 19 [1, 4, 5].
Visual allesthesia may result from interhemispheric transfer of vision from a normal right field to a partially impaired left field $[1,6,7]$ associated with sustained neural activity in the contralateral parietal cortex [8]. This patient had a baseline attentional compromise of left hemi-space, and ongoing seizures shifted the normally attended right hemi-space into the left side. Visual allesthesia, along with palinopsia, differs from epileptic visual illusions and hallucinations from occipital and temporal lesions [9], and implicate a parietal localization.

Conflict of interest statement The authors report no conflicts of interest.

Open Access This article is distributed under the terms of the Creative Commons Attribution Noncommercial License which permits any noncommercial use, distribution, and reproduction in any medium, provided the original author(s) and source are credited. 


\section{References}

1. Jacobs L (1980) Visual allesthesia. Neurology 30:1059-1063

2. Meador KJ, Allen ME, Adams RJ, Loring DW (1991) Allochiria vs. allesthesia. Is there a misperception? Arch Neurol 48:546-549

3. Bender MB, Shapiro MF, Teuber HL (1949) Allesthesia and disturbance of the body scheme. Arch Neurol Psychiatry 62:222235

4. Nakajima M, Yasue M, Kaito N, Kamikubo T, Sakai H (1991) A case of visual allesthesia. No To Shinkei 43:1081-1085

5. Arai T, Irie K, Akiyama M, Kamikubo T, Nakajima M, Sakai H, Abe $\mathrm{T}$ (2002) A case of falcotentorial meningioma with visual allesthesia. No To Shinkei 54:255-259
6. Shewmon A (1981) Visual allesthesia. Neurology 31:496

7. Burcham KJ, Corwin JV, Stoll ML, Reep RL (1997) Disconnection of medial agranular and posterior parietal cortex produces multimodal neglect in rats. Behav Brain Res 86:41-47

8. Schwartz S, Assal F, Valenza N, Seghier ML, Vuilleumier P (2005) Illusory persistence of touch after right parietal damage: neural correlates of tactile awareness. Brain 128:277-290

9. Bien CG, Benninger FO, Urbach H, Schramm J, Kurthen M, Elger CE (2000) Localizing value of epileptic visual auras. Brain 123:244-253 